\title{
Risque et gestion des saignements intraoculaires associés aux anticoagulants oraux
}

Len V. Koh, OD, PhD, FAAO

Optométriste traitant

Mann-Granstaff VA

Medical Center

Chad E. Gosnell, OD, FAAO

Directeur des services

d'optométrie

\author{
Anna R. Well, \\ OD, FAAO \\ Optométriste traitante
}

\begin{abstract}
Résumé
La thrombose veineuse profonde, l'embolie pulmonaire, l'AVC ischémique et l'infarctus du myocarde sont d'importantes maladies thromboemboliques qui touchent des millions de Nord-Américains. La plupart de ces patients sont pris en charge à long terme avec des anticoagulants oraux qui peuvent accroître le risque de saignement, y compris les hémorragies intraoculaires. L'interruption de l'anticoagulation augmente temporairement le risque de thromboembolie et l'anticoagulation continue augmente le risque de saignement; les deux scénarios nuisent à la santé globale du patient. Cet article présente une revue générale et une mise à jour des anticoagulants oraux et traite des façons pour les optométristes de gérer les saignements oculaires associés aux anticoagulants oraux.
\end{abstract}

\section{INTRODUCTION}

La thrombose est la formation de caillots qui nuisent à la circulation sanguine, ce qui entraîne une ischémie ou des dommages aux tissus. Elle peut survenir dans les vaisseaux artériels et veineux. Un thrombus se fragmenter et se détacher pour former un embole, qui passe alors dans la circulation sanguine et finit par se fixer dans un vaisseau sanguin de diamètre plus petit, où il bloque la circulation du sang, y compris dans les yeux, où il peut se manifester en tant qu'embole rétinien (plaque de Hollenhorst) ou occlusion de l'artère rétinienne. ${ }^{1,2}$ Les principales maladies thromboemboliques sont la thrombose veineuse profonde (TVP), l'embolie pulmonaire (EP), l'AVC ischémique et l'infarctus du myocarde (IM). La TVP est la formation de caillots sanguins à l'intérieur des veines profondes, à l'intérieur du corps. Les grandes veines dans le bas de la jambe et la cuisse, le bassin et le bras sont les plus sensibles. Bien que la TVP en soi puisse être bénigne, un embole provenant de la TVP peut se déplacer dans les vaisseaux sanguins et finir par bloquer la circulation sanguine vers les poumons, le cour, le cerveau ou une autre région, ce qui peut causer des lésions tissulaires, une débilité ou la mort. L'EP se définit par l'obstruction d'une artère dans les poumons qui est généralement causée par un embole qui s'est détaché de la TVP. Les causes moins courantes de l'EP comprennent les bulles d'air, les gouttelettes de graisse et les cellules tumorales. Un AVC ischémique se produit lorsqu'un thrombus ou un embole bloque un vaisseau sanguin dans le cerveau. La thrombose cérébrale est une conséquence de l'athérosclérose, tandis que l'embolie cérébrale provient habituellement d'un caillot dans le cœur ou les grandes artères du haut de la poitrine et du cou. De plus, la fibrillation auriculaire augmente la propension de l'embole à voyager et à bloquer la circulation au cerveau, ce qui entraîne un AVC. L'IM (crise cardiaque) se produit lorsque la circulation sanguine d'une artère coronaire vers le cour est soudainement bloquée, causant la mort des cellules myocardiques. Une plaque d'athérome insidieuse dans une artère coronaire présente le risque le plus élevé de crise cardiaque parce que la plaque peut se développer et bloquer la circulation sanguine, ou une rupture de la plaque peut déclencher la formation d'un caillot de sang et bloquer l'approvisionnement en sang du tissu cardiaque. Ensemble, ces maladies thromboemboliques sont responsables de jusqu'à un quart de tous les 
décès dans le monde. ${ }^{1}$ En 2015, 28,4 millions d'Américains adultes ont reçu un diagnostic de maladie cardiaque et 6,5 millions avaient déjà subi un AVC. Par conséquent, les maladies cardiaques et les AVC sont deux des principales causes de décès aux États-Unis, se classant au premier rang (614 348 décès par année) et au cinquième (133 103) respectivement.

L'incidence de la thrombose augmente à mesure que la population vieillit. ${ }^{3}$ Les anticoagulants sont les médicaments de choix pour prévenir et traiter les événements thromboemboliques qui menacent le pronostic vital. Toutefois, les patients qui prennent ces médicaments voient leur risque de saignement augmenter, y compris les hémorragies oculaires. De plus, certaines interventions chirurgicales obligent les patients à changer de médicament ou à cesser l'anticoagulation pendant la période peropératoire. Cet article présente une brève revue générale et une mise à jour des anticoagulants oraux et traite des façons de gérer les saignements oculaires associés aux anticoagulants oraux.

Les fournisseurs de soins oculovisuels rencontrent souvent des patients qui prennent des anticoagulants oraux. Certains de ces patients peuvent se présenter à la clinique avec un saignement intraoculaire, tandis que d'autres peuvent être référés pour une chirurgie oculaire. Nous devons connaître ce qui a trait à ces nouveaux anticoagulants oraux directs (AOD) et savoir comment gérer les deux scénarios. Nous examinerons l'état actuel des connaissances sur le risque d'hémorragie intraoculaire dans les environnements cliniques et chirurgicaux afin d'être mieux préparés à soigner ces patients. Les deux cas cliniques suivants sont présentés pour illustrer des cas qui peuvent se présenter et leur prise en charge.

\section{RAPPORTS DE CAS}

\section{Cas 1 : rivaroxaban (Xarelto)}

Un homme blanc de 70 ans s'est présenté à la clinique de la vue le 12 mai et a déclaré avoir un saignement dans l'œil droit depuis une dizaine de jours. Même si son œil semblait en mauvais état, il ne ressentait ni douleur ni irritation. C'était la première fois que cela arrivait, et il n'avait pas fait d'effort intense ou soulevé quelque chose de lourd avant l'incident. Son dernier examen complet de la vue remontait à deux mois. Il prenait du furoséromide et du métoprolol pour l'hypertension, de l'albutérol-ipratropium pour la maladie pulmonaire obstructive chronique (MPOC) et de la flécaïnide et du rivaroxaban pour la fibrillation auriculaire. Sa tension artérielle récente et son indice de masse corporelle (IMC) étaient respectivement de 168/91 et de 33,4.

Son acuité visuelle habituelle était de 20/25+ OD et à de 20/20- OG avec des erreurs de réfraction de -0,75-1,00x085 OD et -1,25-1,25 x080 OG. Ses pupilles étaient égales et réactives à la lumière, sans déficit pupillaire afférent; le mouvement extraoculaire était complet sans restriction; le champ visuel par confrontation était normal (comptage des doigts). Le test de l'écran était orthophorique. La PIO avec le tonomètre à aplanation de Goldmann était de $18 \mathrm{mmHg}$ OD, $17 \mathrm{mmHg}$ OG @ 1328. L'examen à la lampe à fente a révélé la présence d'une cataracte sclérotique nucléaire de grade 1+ dans les deux yeux et d'une hémorragie sous-conjonctivale du côté droit (figure 1). L'examen du fond d'œil sous pupille dilatée n'avait rien d'anormal avec des rapports C/D de 0,30 dans les deux yeux.

Le patient a été informé du risque de saignement pendant qu'il prenait du rivaroxaban. On lui a donné des larmes artificielles pour la lubrification, et on lui a recommandé d'appliquer une compresse froide q.i.d. et d'éviter de faire des efforts intenses ou de soulever des objets lourds. On lui a expliqué que l'hémorragie se résorberait au cours des prochaines semaines et qu'il devrait revenir à la clinique après quelques semaines, à moins que l'hémorragie et sa vision ne s'aggravent, auquel cas il devrait revenir plus tôt. Son dispensateur de soins primaires a été informé de l'hémorragie oculaire. La dernière analyse des paramètres de coagulation datant du 16 février 2016 a révélé un RIN de 1,9 (réf. 0,8-1,14) et un TP de 21,3 (réf. 11,8-13,6). L'hémorragie sous-conjonctivale à l'œil droit s'est résorbée au cours des semaines suivantes et on a dit au patient de continuer le traitement de soutien et de retourner à la clinique après un an pour un examen complet de la vue.

\section{Cas 2 : warfarine (Coumadin)}

Un homme blanc de 77 ans s'est présenté à la clinique le 26 octobre et a signalé un problème de vision floue à évolution lente de près et de loin avec ses verres bifocaux habituels. Il a déclaré ne pas avoir d'éblouissements, de corps flottants, de perte de vision et de douleur oculaire. Son dernier examen de la vue remontait à l'année précédente. Il prenait du citalopram pour la dépression, de la ranitidine pour le reflux gastro-œesophagien, du carvédilol et de la spironolactone pour l'hypertension, et de la warfarine pour l'insuffisance de la valve aortique et la fibrillation auriculaire chronique. Sa TA et son IMC étaient respectivement de 127/86 et de 27 au dernier examen.

Son acuité visuelle habituelle était de 20/40-2 PH 20/30-2 OD et de 20/30-2 PHNI OG avec des erreurs de réfraction de $+0,75-1,00 \times 070$ OD et +1,75-1,00x105 OG. Ses pupilles étaient égales et réactives à la lumière sans déficit pupillaire afférent; le mouvement extraoculaire était complet sans restriction; le champ visuel par confrontation était normal 
(comptage des doigts). Le test de l'écran était orthophorique. La PIO avec le tonomètre à aplanation de Goldmann était de 15 mmHg OD, 16 mmHg OG @ 0730. L'examen à la lampe à fente a révélé une cataracte mixte de grade 2+ OU. L'examen du fond d'œil sous pupille dilatée a révélé des rapports C/D de 0,20 OD et 0,25 OG. La rétine périphérique présentait une grande zone d'hémorragie choroïdienne avec des exsudats annulaires curvilignes OG (figure 2).

Figure 1 : hémorragie sous-conjonctivale à l'œil droit

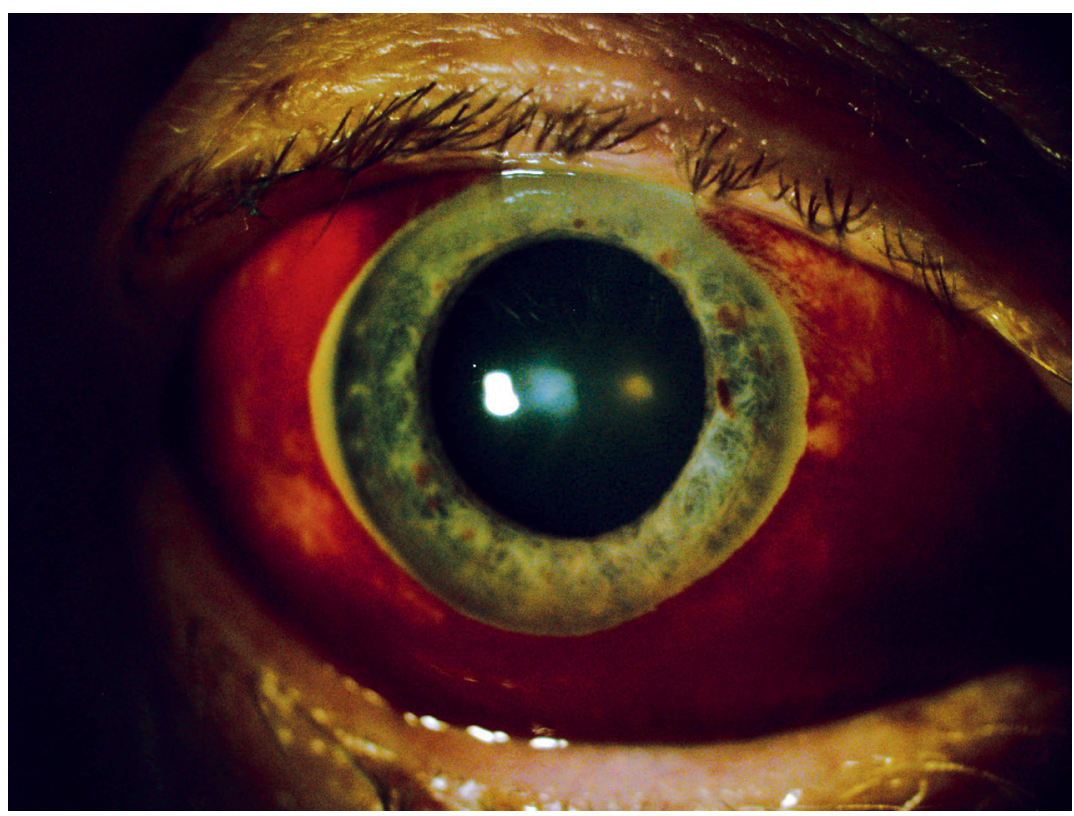

Figure 2 : hémorragie chorö̈dienne avec des exsudats annulaires curvilignes à l'œil gauche

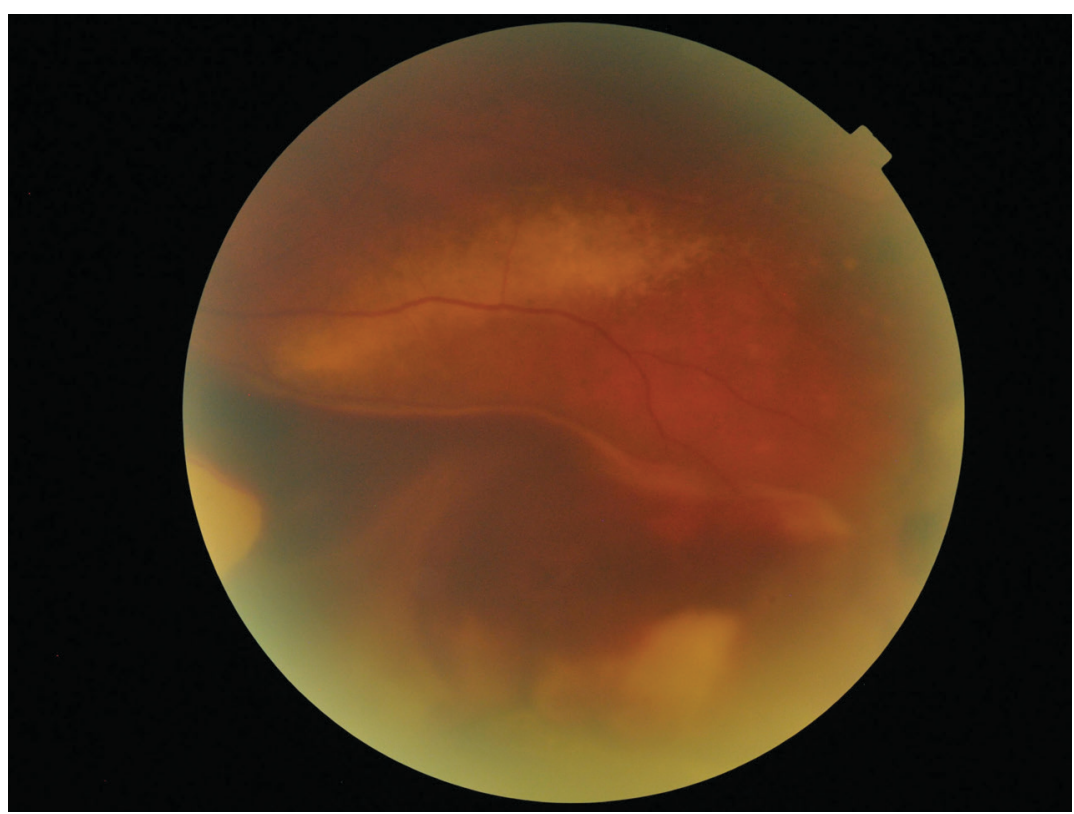


Le patient a été aiguillé vers un spécialiste local de la rétine pour un examen et une prise en charge plus poussés. Il a reçu un diagnostic de choriorétinopathie exsudative hémorragique périphérique (CREHP) et a été traité avec des injections anti-VEGF par le spécialiste de la rétine. L'équipe assurant le suivi de son traitement anticoagulant a été informée de la découverte oculaire. Toutefois, le spécialiste de la rétine n'a pas recommandé l'abandon de l'anticoagulant oral, puisqu'il peut s'agir d'une coïncidence fortuite et non d'une cause de la CREHP. On a recommandé au patient de revenir pour les soins oculovisuels habituels après la prise en charge des troubles rétiniens par le spécialiste de la rétine. L'examen du dossier du patient a confirmé qu'il prenait la moitié d'un comprimé de warfarine (2,5 mg) par la bouche au coucher, mais qu'il avait pris un comprimé complet le dimanche, le mardi et le jeudi. Les résultats des analyses des paramètres d'anticoagulation récents sont présentés au tableau 1.

Tableau 1 : Surveillance régulière du RIN et du TP chez un patient qui prenait de la warfarine (cas 2)

\begin{tabular}{|l|l|l|}
\hline DATE & RIN & TP \\
\hline 16 MAI & 2,3 (réf. 0,8-1,14) & 25,4 (réf. 12-14,9) \\
\hline 21 JUIN & 2,3 & 25,8 \\
\hline 30 JUIN & 2,2 & 25,2 \\
\hline 26 JUILLET & 2,4 & 26,2 \\
\hline 23 AOÛT & 2,6 & 28,1 \\
\hline 27 SEPT & 1,6 & 19,5 \\
\hline 13 OCT & 2,5 & 27,6 \\
\hline 14 NOV & 1,8 & 21,3 \\
\hline
\end{tabular}

\section{DISCUSSION}

\section{Processus de coagulation}

Les protéases, les cofacteurs et les inhibiteurs de protéase présents dans le plasma participent à des interactions complexes, régulées séquentiellement, pour générer de la fibrine, ce qui conduit à la formation d'un bouchon plaquettaire ou thrombus. Ce thrombus est le produit final du processus de coagulation sanguine et est composé de plaquettes activées et d'une protéine fibreuse, la fibrine (facteur Ia). ${ }^{4}$ En bref, la cascade de coagulation sanguine peut être initiée par une voie intrinsèque ou une voie extrinsèque. La voie intrinsèque est activée par des dommages internes au vaisseau sanguin qui exposent le collagène à des plaquettes en circulation. Elle est plus lente que la voie extrinsèque et implique les facteurs XII, XI, IX et VIII. La voie extrinsèque est activée par un traumatisme externe qui compromet l'intégrité de la paroi du vaisseau sanguin et cause une fuite du sang. Elle fait intervenir les facteurs III et VII. Les deux voies convergent vers la voie commune en activant le facteur X. Dans la voie commune, la prothrombine (facteur II) est activée en thrombine (facteur IIa) et le fibrinogène (facteur I) en fibrine, qui « colle » les plaquettes à l'aide de facteur XIII pour stabiliser le caillot (Figure 3). ${ }^{5}$ Cette compréhension du processus de coagulation fournit une bonne base pour comprendre les mécanismes d'action des anticoagulants oraux.

\section{Classes d'anticoagulants}

La coagulation sanguine ou la formation de caillots dépend des interactions entre plusieurs facteurs de coagulation et les plaquettes. Cette cascade complexe offre diverses cibles thérapeutiques pour réguler et atténuer la thrombose. À l'heure actuelle, il existe trois catégories principales de médicaments antithrombotiques pour traiter les caillots. Les anticoagulants comprennent l'héparine non fractionnée (HNF), l'héparine à faible poids moléculaire (HFPM), la warfarine et les anticoagulants oraux directs (AOD). Ils sont administrés par voie sous-cutanée et habituellement, pour les patients à risque de thrombus, dans le sang à écoulement lent des veines. ${ }^{6}$ Les antiplaquettaires oraux comprennent l'aspirine, le clopidogrel (Plavix) et le dipyridamole (Persatine). Ils sont prescrits le plus souvent aux patients qui présentent un risque de caillots provenant du système artériel, où la circulation est rapide. L'aspirine bloque irréversiblement l'enzyme cyclooxygénase et réduit la production de thromboxane A2, un puissant stimulateur de l'agrégation plaquettaire. Le clopidogrel inhibe de façon sélective la fixation de l'adénosine diphosphate (ADP) à son récepteur plaquettaire P2Y12, et donc l'activation du complexe GPIIb/IIIa provoquée par l'ADP, de sorte que l'agrégation pla- 
quettaire est inhibée. Le dipyridamole inhibe l'activité de l'adénosine désaminase et de la phosphodiestérase, ce qui provoque l'accumulation de médiateurs antiplaquettaires comme l'adénosine, les nucléotides d'adénine et l'AMP cyclique. ${ }^{7}$ Les médicaments thrombolytiques ou fibrinolytiques comprennent l'altéplase (TPA), le rétéplase (Retavase) et la streptokinase (Streptase). On les administre par voie intraveineuse à l'urgence pour dissoudre les caillots préexistants dans le poumon, le cœur ou le cerveau. L'altéplase déclenche la fibrinolyse locale en se liant à la fibrine, ce qui induit la conversion du plasminogène en plasmine et entraîne la dissolution du caillot de fibrine. Le rétéplase est un activateur du plasminogène recombinant qui a une demi-vie plus longue que l'altéplase. La streptokinase est un polypeptide à chaîne unique dérivé des cultures de streptocoque bêta hémolytique. Elle se lie au plasminogène et le convertit en plasmine active pour dégrader les caillots de fibrine. Cet article porte principalement sur les anticoagulants oraux, y compris les AOD, mais mentionne également d'autres classes d'antithrombotiques.

Figure 3: Cascade de coagulation et cibles des anticoagulants oraux4. FXa indique le facteur Xa; HK, kininogène à poids moléculaire élevé ; $P K$, prékallekreine; PL, phospholipide; et TF, facteur tissulaire.

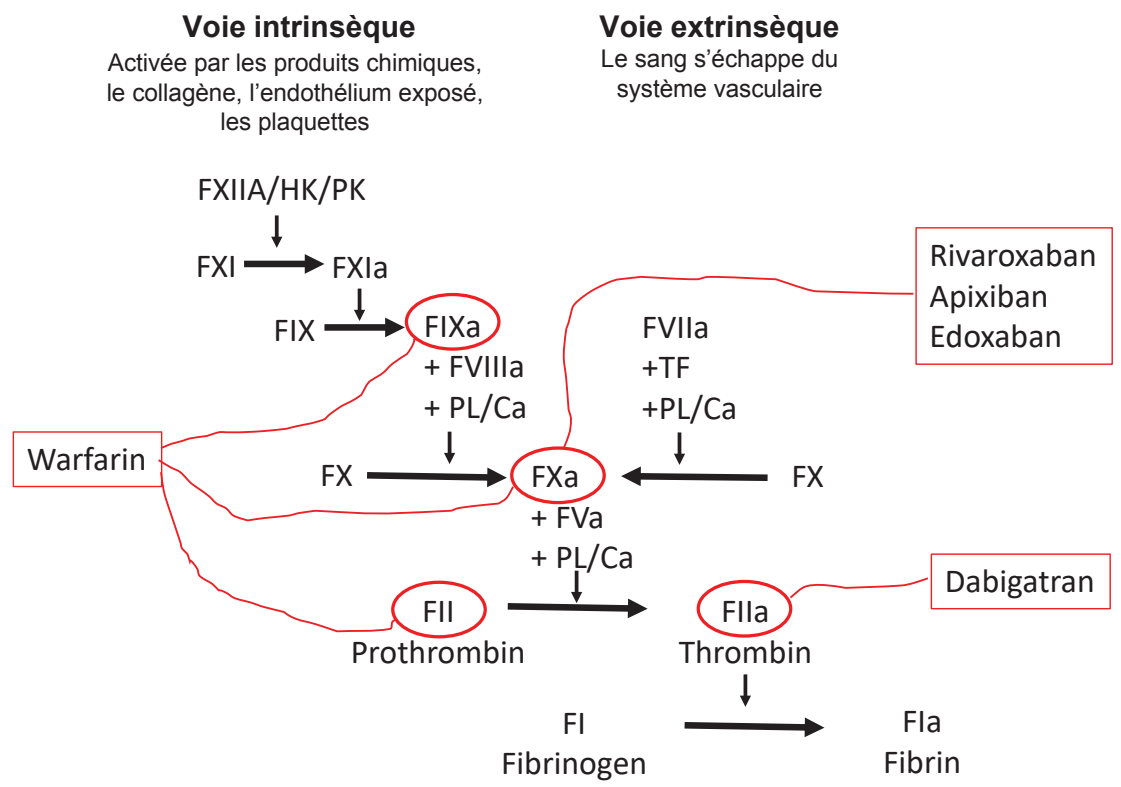

\section{Antagonistes de la vitamine K (AVK)}

La warfarine (Coumadin), l'AVK le plus couramment prescrit, a été approuvée en 1955 pour le traitement des complications thromboemboliques associées à la fibrillation auriculaire. Elle est encore communément associée au poison à rat parce qu'elle a été formulée à l'origine avec une base de maïs comme poison à rat efficace dans les fermes. Le président Dwight D. Eisenhower a été l'un des premiers patients à recevoir de la warfarine lorsqu'il a subi une crise cardiaque pendant ses vacances en 1955. Pendant plus d'un demi-siècle, la warfarine est demeurée le traitement de base pour l'anticoagulation à long terme et la prévention de diverses maladies thromboemboliques. Les anticoagulants dérivés de la coumarine, y compris l'acénocoumarol, la phénprocoumone et la fluindione, sont d'autres AVK. Ils sont rarement utilisés aux États-Unis et ne sont donc mentionnés qu'à titre informatif. La warfarine pose des défis cliniques en raison de sa fenêtre thérapeutique étroite et ses diverses interactions médicamenteuses. Il est difficile et long d'ajuster la posologie de façon à maintenir les patients dans la fenêtre thérapeutique sécuritaire, ce qui exige d'équilibrer le risque de thrombose d'un côté et le risque de saignement de l'autre. Les avantages de la warfarine sont que son utilisation est appuyée par un vaste corpus de données cliniques, qu'elle est peu coûteuse et qu'il y a diverses façons d'inverser ses effets anticoagulants, comme la vitamine K, le plasma frais congelé (PFC) et le concentré de complexe prothrombique (CCP). Les analyses sanguines nécessaires avant le début du traitement par warfarine comprennent le temps de prothrombine (TP) avec un rapport international normalisé (RIN) et le temps de céphaline activé (TCA), un hémogramme complet, un dosage de la créatinine sérique, des tests de fonction hépatique et un test de grossesse pour les femmes en âge de procréer. ${ }^{8}$ 
Le RIN a été proposé en 1985 comme mesure standard pour la surveillance du traitement par warfarine. Il permet d'éviter les différences interlaboratoires causées par l'utilisation de thromboplastines à sensibilité différente dans les résultats d'essai. Le RIN est calculé en mettant le rapport de temps de prothrombine (temps de prothrombine du patient divisé par un temps de prothrombine témoin) à la puissance d'un coefficient appelé indice de sensibilité international (ISI). ${ }^{9}$ La dose quotidienne initiale recommandée est de $5 \mathrm{mg}$ pendant les deux premiers jours, et est ajustée en fonction du TP et du RIN à partir du troisième jour. La dose d'entretien varie de $<2 \mathrm{mg}$ à $\geq 10 \mathrm{mg}$ par jour. La valeur cible habituelle du RIN est de l'ordre de 2,0 à 3,0 chez les patients qui ont une thromboembolie veineuse, et un peu plus élevée chez les patients qui ont des valves cardiaques mécaniques. L'intervalle de surveillance va d'une journée chez les patients hospitalisés à 12 semaines chez les patients les plus stables. Il faut avertir les patients qui prennent de la warfarine qu'ils doivent retourner à la clinique lorsqu'une complication de saignement survient, comme des contusions inexpliquées, des saignements de nez et du sang dans l'urine ou les selles. De plus, certains aliments et médicaments peuvent accroître ou diminuer l'efficacité de la warfarine. Par exemple, les épinards, le chou frisé et les feuilles de « chou vert » contiennent des teneurs élevées de vitamine K et peuvent réduire l'efficacité de la warfarine. ${ }^{10}$ Perle clinique : il faut faire preuve de prudence lorsque l'on recommande aux patients atteints de dégénérescence maculaire liée à l'âge qui prennent de la warfarine de manger davantage de légumes à feuilles vertes, puisque cela pourrait affecter l'efficacité de l'anticoagulation et augmenter le risque de thrombose.

\section{Anticoagulants oraux directs (AOD)}

Une nouvelle vague d'anticoagulants oraux est apparue au cours de la dernière décennie. Ils permettent d'éviter les ajustements posologiques et les analyses de suivi régulières qui sont nécessaires avec la warfarine. Cette classe de médicaments est également appelée anticoagulants oraux spécifiques à une cible, inhibiteurs directs oraux (IDO), nouveaux anticoagulants oraux (NACO) et anticoagulants oraux non antagonistes de la vitamine K. ${ }^{11}$ Ils peuvent être classés comme inhibiteurs directs de la thrombine (facteur IIa) (IDT) : dabigatran (Pradaxa) ou inhibiteurs directs du facteur Xa : rivaroxaban (Xarelto), apixaban (Eliquis), édoxaban (Savaysa) et bétrixaban (Bevyxxa). Vous remarquerez que les noms génériques de ces derniers agents se terminent tous par «Xa-ban». Ces nouveaux agents permettent un dosage fixe et une surveillance peu fréquente, et sont devenus des substituts populaires de la warfarine. Néanmoins, la warfarine est encore largement utilisée parce qu'il n'existe pas d'antidote efficace et d'expérience clinique pour ces nouveaux AOD et que leur coût est plus élevé. ${ }^{12}$ Dans l'ensemble, les cliniciens disposent maintenant d'un arsenal beaucoup plus important pour la prise en charge chronique des maladies thromboemboliques. En général, il est nécessaire d'évaluer et de documenter l'état de la coagulation au moyen d'essais en laboratoire comme la numération plaquettaire, le temps de prothrombine (TP) et le temps de céphaline activé (TCA) avant de commencer un traitement anticoagulant avec la warfarine ou les AOD. La mesure de la créatinine sérique et des analyses de la fonction hépatique sont également effectuées pour ajuster la dose en cas d'insuffisance rénale ou hépatique. Curieusement, la surveillance de routine en laboratoire du temps de coagulation n'est pas nécessaire pour les AOD, à moins de surdose présumée ou de saignements manifestes.

Le dabigatran etexilate (Pradaxa, 220-300 mg/j), le seul IDT oral, est un promédicament administré par voie orale et converti en dabigatran dans le foie qui inhibe la thrombine libre et la thrombine liée à la fibrine. Il est susceptible de se décomposer s'il est exposé à l'humidité et est donc emballé avec un dessiccatif. Les comprimés doivent être consommés dans un délai de quatre mois pour garantir une efficacité optimale. Le dabigatran est utilisé pour la prévention et la prise en charge de la TVP et pour prévenir les AVC chez les patients atteints de fibrillation auriculaire. Il ne doit pas être utilisé chez les patients qui ont des valvules cardiaques prothétiques ou pendant la grossesse. Récemment, l'idarucizumab (Praxbind) a été approuvé pour inverser l'effet anticoagulant du dabigatran en cas de saignements menaçant le pronostic vital. Il est administré par voie intraveineuse en 2 doses distinctes de 2,5 g, à un intervalle de temps d'au plus 15 minutes. ${ }^{12}$

Le rivaroxaban (Xarelto, 10-30 mg/j), l'apixaban (Eliquis, 5-10 mg/j), l'édoxaban (Savaysa, 30-60 mg/j) et le bétrixaban (Bevyxxa, 80-160 mg/j) sont des inhibiteurs directs du facteur Xa qui empêchent la conversion de la prothrombine en thrombine. Ils se lient directement au facteur Xa plutôt que d'accroître l'activité de l'antithrombine médiée par sa liaison à l'héparine. Ils sont indiqués pour la prévention et le traitement de la TVP et pour la prévention des AVC chez les patients atteints de fibrillation auriculaire. La posologie des médicaments de ce groupe varie selon l'indication clinique et la fonction rénale du patient. Le rivaroxaban est le seul médicament de cette catégorie qui doit être pris avec des aliments pour avoir une efficacité optimale. Il n'existe pas d'antidote spécifique à ces inhibiteurs directs du facteur Xa. En cas de saignement majeur ou menaçant le pronostic vital, on utilise des méthodes d'inversion générales comme l'administration d'un antifibrinolytique (acide tranexamique, acide epsilon-aminocaproïque) ou la prise de charbon actif par voie orale pour éliminer les médicaments non absorbés du tractus gastrointestinal et on interrompt le traitement anticoagulant (tableau 2). ${ }^{12}$ 
Tableau 2 : Avantages et inconvénients des AOD par rapport à la warfarine. ${ }^{8}$

\begin{tabular}{|l|l|l|}
\hline & Warfarine & $\begin{array}{l}\text { AOD } \\
\text { (Dabigatran, rivaroxaban, apixaban, édoxaban) }\end{array}$ \\
\hline Posologie & Une fois par jour & Peut nécessiter une prise plus fréquente \\
\hline Restrictions alimentaires & Surveiller l'apport en vitamine K & Prendre le rivaroxaban avec de la nourriture \\
\hline Surveillance & Surveillance régulière TP/RIN & Non requise \\
\hline $\begin{array}{l}\text { Interactions } \\
\text { médicamenteuses }\end{array}$ & Nombreuses & $\begin{array}{l}\text { Inhibiteurs du CYP-34A et modulateurs de la } \\
\text { p-glycoprotéine }\end{array}$ \\
\hline $\begin{array}{l}\text { Temps dans la plage } \\
\text { thérapeutique }\end{array}$ & $\sim 65 \%$ & $>65 \%$ \\
\hline Agents d'inversion & $\begin{array}{l}\text { Vitamine K, PFC, CCP, FVIIa } \\
\text { recombinant }\end{array}$ & $\begin{array}{l}\text { Lidarucizumab renverse l'effet du dabigatran. Le } \\
\text { charbon activé; les agents antifibrinolytiques; le } \\
\text { concentré de complexe prothrombique peut être utilisé } \\
\text { pour les saignements mettant le pronostic vital en } \\
\text { danger avec d'autres AOD. }\end{array}$ \\
\hline
\end{tabular}

AOD, anticoagulant oral direct; TP, temps de prothrombine; RIN, rapport international normalisé; PFC, plasma frais congelé; PCC, concentré de complexe prothrombique; rFVIIa, facteur VII activé recombinant

\section{Effets indésirables oculaires des AOD}

Récemment, de nombreux cliniciens se sont tournés vers les AOD comme médicaments de choix pour la prophylaxie et le traitement des maladies thromboemboliques. De nombreuses études ont été réalisées pour évaluer leur propension à provoquer des saignements majeurs par rapport à la warfarine, et les données probantes cumulées de multiples essais cliniques et d'autres études post-commercialisation ont montré que les AOD ont une efficacité antithrombotique égale ou supérieure à celle de la warfarine et qu'ils sont associés à un risque plus faible d'hémorragie intracrânienne. ${ }^{13}$ On en connaît moins sur le risque d'hémorragie intraoculaire associé aux AOD comparativement à celui de la warfarine. Toutefois, une méta-analyse récente de 12 essais portant sur 102627 patients a fourni des données probantes aux fournisseurs de soins oculovisuels. On a constaté que les AOD réduisaient le risque de saignement intraoculaire d'environ $22 \%$ comparativement à la warfarine chez les patients atteints de fibrillation auriculaire ou de thromboembolie veineuse. ${ }^{14}$

Bien que le mécanisme exact par lequel les AOD permettent de réduire le risque d'hémorragie intraoculaire comparativement à la warfarine ne soit pas clair, ils sont probablement plus sûrs, car ils ne ciblent qu'un seul site dans la cascade de coagulation plutôt que de multiples sites, comme c'est le cas avec la warfarine. ${ }^{13}$ Ces résultats sont particulièrement importants pour les patients présentant un risque de saignement oculaire élevé au départ, comme dans le cas de dégénérescence maculaire liée à l'âge (DMLA) exsudative et d'autres formes de néovascularisation choroïdienne. Les patients atteints de DMLA sont 10 fois plus susceptibles de souffrir d'hémorragie intraoculaire massive et de voir leur pronostic visuel s'aggraver s'ils prennent des anticoagulants, ${ }^{15}$ tel qu'illustré ci-dessus dans le cas 2.

On devrait évaluer avec soin le risque plus élevé d'hémorragie par rapport aux bienfaits possibles avant d'administrer des anticoagulants, en particulier en contexte peropératoire. On estime qu'environ $10 \%$ des patients recevant des anticoagulants doivent interrompre ce traitement en cas d'intervention chirurgicale. ${ }^{16} \mathrm{Il}$ est important que les fournisseurs de soins oculovisuels sachent s'il faut interrompre l'anticoagulation avant d'aiguiller les patients pour des interventions ophtalmiques. Les complications hémorragiques chez les patients anticoagulés qui subissent une chirurgie ophtalmique incluent la présence de sang dans les larmes, l'hyphème et des hémorragies vitréennes, sous-conjonctivales, sous-rétiniennes et choroïdiennes. ${ }^{17}$ Heureusement, le risque d'hémorragie grave menaçant la vue lors d'interventions ophtalmiques de routine chez les patients sous anticoagulants est relativement faible, et donc l'arrêt des anticoagulants oraux n'est pas recommandé. ${ }^{18}$ Dans l'étude RE-LY (Randomized Evaluation of Long-term Anticoagulation therapy), aucun saignement significatif n'a été observé chez les patients prenant de la warfarine ou du dabigatran qui ont subi une opération de la cataracte. ${ }^{19}$ De plus, aucun risque accru de complications peropératoires n'a été observé dans une petite étude menée auprès de 36 patients sous anticoagulants ayant subi une chirurgie vitréorétinienne. À l'opposé, les procédures oculoplastiques à haut risque, telles que la dacryocystorhinostomie, la chirurgie orbitale profonde et la chirurgie des paupières majeure, nécessitent l'arrêt du traitement par AOD 48 heures avant l'intervention. ${ }^{20}$ Bien qu'il n'existe actuellement aucune directive relative à l'arrêt des AOD avant une chirurgie ophtalmique, de l'avis général, les AOD peuvent être interrompus de 
manière transitoire sans risque chez les patients à risque thrombotique faible à moyen sans nécessiter de traitement de substitution, alors que chez les patients à risque élevé, on devrait évaluer avec soin les risques de thromboembolie par rapport au risque généralement faible d'hémorragie oculaire menaçant la vue (tableau 3). ${ }^{21}$

Table 3 : Pre- and post-operative management of patients taking $D O A C s^{8,17}$

\begin{tabular}{|l|l|l|l|l|}
\hline Médicament & Mode d'action & Demi-vie, fréquence & Chirurgie mineure & Chirurgie majeure \\
\hline Apixaban & $\begin{array}{l}\text { inhibiteur du } \\
\text { facteur Xa }\end{array}$ & 12 h, b.i.d. & $\begin{array}{l}\text { Arrêt 2 jours avant } \\
\text { Reprendre } 1 \text { jour après }\end{array}$ & $\begin{array}{l}\text { Arrêt } 3 \text { jours avant } \\
\text { Reprendre 2 jours après }\end{array}$ \\
\hline $\begin{array}{l}\text { Dabigatran } \\
\text { (promédicament) }\end{array}$ & $\begin{array}{l}\text { Inhibiteur direct } \\
\text { de la thrombine } \\
\text { (facteur IIa) }\end{array}$ & 12-17 h, b.i.d. & $\begin{array}{l}\text { Arrêt 2 jours avant } \\
\text { Reprendre } 1 \text { jour après }\end{array}$ & $\begin{array}{l}\text { Arrêt 3 jours avant } \\
\text { Reprendre 2 jours après }\end{array}$ \\
\hline Édoxaban & $\begin{array}{l}\text { Inhibiteur du } \\
\text { facteur Xa }\end{array}$ & 9-11 h, q.i.d. & $\begin{array}{l}\text { Arrêt 2 jours avant } \\
\text { Reprendre } 1 \text { jour après }\end{array}$ & $\begin{array}{l}\text { Arrêt } 3 \text { jours avant } \\
\text { Reprendre 2 jours après }\end{array}$ \\
\hline $\begin{array}{l}\text { Rivaroxaban } \\
\text { prendre avec de } \\
\text { la nourriture) }\end{array}$ & $\begin{array}{l}\text { Inhibiteur du } \\
\text { facteur Xa }\end{array}$ & $5-9$ h, q.i.d. & $\begin{array}{l}\text { Arrêt 2 jours avant } \\
\text { Reprendre } 1 \text { jour après }\end{array}$ & $\begin{array}{l}\text { Arrêt 3 jours avant } \\
\text { Reprendre 2 jours après }\end{array}$ \\
\hline Warfarine & $\begin{array}{l}\text { Synthèse } \\
\text { des facteurs } \\
\text { dépendant de la } \\
\text { vitamine K }\end{array}$ & $36-42$ h, q.i.d. & $\begin{array}{l}5 \text { jours pour obtenir } \\
\text { plage thérapeutique }\end{array}$ & $\begin{array}{l}5 \text { jours pour obtenir un } \\
\text { RIN } \leq 1,4 \text { ou dans la plage } \\
\text { thérapeutique }\end{array}$ \\
\hline
\end{tabular}

AOD, anticoagulant oral direct; MA, mécanisme d'action; RIN, rapport international normalisé

\section{Éducation des patients}

Les anticoagulants sont utilisés pour prévenir la formation de caillots sanguins observée dans les cas de fibrillation auriculaire (qui peut provoquer une accumulation de sang dans les cavités supérieures du cœur), de remplacement valvulaire (où des caillots sanguins peuvent se former sur ou près de la valve cardiaque), de dysfonctionnement ventriculaire gauche ou de cardiomyopathie (qui peut causer l'accumulation de sang dans les cavités inférieures du cœur), de thrombose veineuse profonde, d'embolie pulmonaire, d'AVC, d'accident ischémique transitoire (qui est souvent le signe précoce d'un futur AVC) et après une intervention chirurgicale qui augmente le risque de formation de caillots sanguins.

Des visites régulières à la clinique d'anticoagulation sont importantes pour surveiller le RIN chez les patients prenant de la warfarine. L'équipe responsable du suivi de l'anticoagulation comprend un médecin, une infirmière, un pharmacien et des membres du laboratoire. Si un patient est atteint de fièvre, d'infection, de diarrhée ou de vomissements, l'équipe doit en être informée, puisque cela peut affecter la valeur du RIN. Les médicaments, les aliments, les herbes, les vitamines et l'alcool peuvent interagir avec les anticoagulants. L'équipe peut recommander au patient d'éviter les sports de contact et les exercices qui peuvent entraîner un risque accru de chute et de blessure.

Les patientes qui sont enceintes ou prévoient le devenir doivent communiquer immédiatement avec leur équipe d'anticoagulation parce que le traitement par warfarine comporte de graves risques pour le foetus, surtout pendant le premier trimestre.

\section{Perles cliniques}

Les AOD peuvent désactiver les facteurs de coagulation activés libres ou liés au caillot. Ils ne nécessitent pas de suivi fréquent de l'effet anticoagulant, car leur efficacité est plus prévisible pour une dose donnée. Bien que le risque global de saignement soit comparable à celui de la warfarine, les AOD présentent un risque plus faible de saignement intracrânien. Toutefois, les AOD coûtent cher, et il est plus difficile de surveiller l'observance thérapeutique du patient qu'avec la warfarine.

Les AOD sont contre-indiqués chez les patients souffrant d'insuffisance rénale sévère, ceux qui ont des valvules cardiaques prothétiques et pendant la grossesse. Ces agents ne sont généralement pas administrés aux personnes dont l'indice de masse corporelle (IMC) est supérieur à $40 \mathrm{~kg} / \mathrm{m}^{2}$ ou dont le poids est supérieur à $120 \mathrm{~kg}$. 
Les AOD sont généralement administrés à des doses fixes sans surveillance en laboratoire, mais des tests de laboratoire semblables à ceux qui sont réalisés avant le début d'un traitement par warfarine doivent être effectués avant l'administration de ces agents.

L'interruption de l'anticoagulation augmente temporairement le risque de thromboembolie et l'anticoagulation continue augmente le risque de saignement; les deux scénarios nuisent à la santé globale du patient. Les fournisseurs de soins oculovisuels doivent travailler en étroite collaboration avec les fournisseurs de soins primaires et/ou l'équipe d'anticoagulation pour décider de la meilleure ligne de conduite à adopter pour chaque cas de saignement oculaire. $\bullet$

\author{
AUTEUR-RESSOURCE \\ Len V. Koh O.D., Ph. D., FAAO \\ Optométriste traitant \\ Mann-Granstaff VA \\ Medical Center \\ Spokane, Washington 99208 \\ len.koh@va.gov \\ Cellulaire : 509-434-7032 \\ Télécopieur : 509-434-7132
}

\title{
RÉFÉRENCES
}

1. Goldhaber S.Z., Bounameaux H. Pulmonary embolism and deep vein thrombosis. Lancet 2012; 379(10027): 1835-46.

2. Zimmerman L.E. Embolism of central retinal artery: secondary to myocardial infarction with mural thrombosis. Arch Ophthalmol 1965;73:822-6.

3. Wendelboe, A.M., Raskob, G.E. 2007. Global burden of thrombosis: epidemiologic aspects. Circ Res 2016;118:1340-7.

4. Davie, E.W., K. Fujikawa et W. Kisiel. The coagulation cascade : initiation, maintenance, and regulation. Biochemistry 1991; 30(43):10363-1070.

5. Hoffman M., Monroe D.M. Impact of non-vitamin K antagonist oral anticoagulants from a basic science perspective. Arterioscler Thromb Vasc Biol 2017;37:1812-8.

6. Bickman, J.K., Baglin, T., Meijers, J.M., Renne, T. Novel targets for anticoagulants lacking bleeding risk. Curr Opin Hematol 2017;24:419-26.

7. Sozeri Y. and Salim S. Anticlotting agents and the surgical management of glaucoma. Curr Opin Ophthalmol 2018;29:185-9.

8. Hirsh J. Oral anticoagulants : mechanism of action, clinical effectiveness, and optimal therapeutic range. Chest 2001;119(1):8S-21S.

9. ICSH/ICTH recommendations for reporting prothrombin time in oral anticoagulant control. International Committee for Standardization in Haematology and International Committee on Thrombosis and Haemostasis. $J$ Clin Pathol 1985;38:133-4.

10. Wells P.S., Holbrook A.M., Crowther R.R., Hirsh J. Interactions of warfarin with drugs and food. Ann Intern Med 1994;121(9):676-83.

11. Barnes, G.D., Ageno, W., Ansell, J., Kaatz, S. Recommendation on the nomenclature for oral anticoagulants : communication from the SSC of the ISTH. $J$ Thromb Haemost 2015;13(6):1154-6.

12. Hinojar R., Jimerez-Natcher J.J., Fernandez-Golfin C., Zamorano J.L. New oral anticoagulants: a practical guide for physicians. Eur Heart J Cardiovasc Pharmacother 2015;1:134-45.
13. Monaco, L., Biagi, C., Conti, V., et coll. Safety profile of DOACs. Br J Clin Pharmacol 2017;83:1532-43.

14. Sun M.T., Wood M.K., Chan W. et coll. Risk of intraocular bleeding with novel oral anticoagulants compared with warfarin- a systemic review and meta-analysis. JAMA Ophthalmol 2017;135(8):864-87.

15. Tilanus, M.A., Vaandrager, W., Cuypers, M.H., Verbeek, A.M., Hoyng, C.B. Relationship between anticoagulant medication and massive intraocular hemorrhage in age-related macular degeneration. Graefes Arch Clin Exp Ophthalmol 2000;238(6):482-5.

16. Douketis J.D., Berger P.B., Dunn A.S., et coll; American College of Chest Physicians. The perioperative management of antithrombotic therapy: American College of Chest Physicians EvidenceBased Clinical Practice Guidelines (8e édition). Chest 2008;133(6 suppl):299S-339S

17. Talany, G., Guo, M., Etminan, M. Risk of intraocular hemorrhage with new oral anticoagulants. Eye 2017;31:628-31.

18. Kong K.L., Khan J. Ophthalmic patients on antithrombotic drugs : a review and guide to perioperative management. Br J Ophthalmol 2015;99(8):1025-30.

19. Healey, J.S., Eikelboom, J., Douketis, J., et coll.; RE-LY Investigators. Periprocedural bleeding and thromboembolic events with dabigatran compared with warfarin: results from the Randomized Evaluation of Long-term Anticoagulation Therapy (RE-LY) randomized trial. Circulation 2012;126(3):343-8.

20. Esparaz E.S., Sobel R.K. Perioperative management of anticoagulants and antiplatelet agents in oculoplastic surgery. Curr Opin Ophthalmol 2015;26(5):422-8.

21. Patel R., Charles S., Jalil A. Antiplatelets and anticoagulants in vitreoretinal surgery, with a special emphasis on novel anticoagulants: a national survey and review. Graefes Arch Clin Exp Ophthalmol 2017;255:1275-85. 